\title{
ОПТИМАЛЬНОЕ УПРАВЛЕНИЕ ПАРАЛЛЕЛЬНЫМИ ВЕНТИЛЯТОРНЫМИ УСТАНОВКАМИ НА ОСНОВЕ НЕЧЕТКОЙ ЛОГИКИ ВТОРОГО ПОРЯДКА
}

\author{
А.В. КАШНИКОВ \\ Горный институт УрО РАН, г. Пермь
}

\begin{abstract}
Аннотация: Решение задачи оптимального управления проветриванием при наличии нескольких вентиляторных установок предполагает необходимость определения, в какой степени каждый из вентиляторов должен изменять частоту своего вращения для обеспечения заданных расходов воздуха на регуляторах на каждом шаге управления. При этом необходимо учитывать не только степень влияния вентилятора на конкретный регулятор, но и его текущую частоту вращения. Для учета данных факторов предлагается использование нечетких множеств второго порядка интервального типа для определения входной переменной недостатка/избытка воздуха, в которых функции принадлежности определяют границы оптимальности для каждого вентилятора. Также в статье вводится понятие зоны влияния вентилятора, используемое для обобщения требования наличия полностью открытого регулятора на случай нескольких вентиляторных установок. Приведены результаты моделирования работы алгоритма управления и их сравнение с применением подхода, основанного на нечеткой логике первого порядка.
\end{abstract}

Ключевые слова: нечеткие множества второго порядка интервального типа, вентилятор, автоматический регулятор, ПИД-регулирование, система автоматического управления проветриванием.

Внедрение систем автоматического управления проветриванием (САУП) на рудниках горнодобывающих предприятий позволяет существенно снизить эксплуатационные затраты и повысить безопасность ведения горных работ за счет точного распределения воздуха по рабочим зонам [1,5].

САУП включает в себя одну или несколько главных вентиляторных установок и автоматические регуляторы воздуха $[3,7,8]$.

Как было показано в работе [2], реализация подобных систем на основе ПИДрегулирования обладает существенными недостатками, в связи с чем было предложено использование аппарата нечеткой логики для управления элементами САУП (для случая одной главной вентиляторной установки).

При управлении сложными вентиляционными системами, в которых проветривание обеспечивается несколькими главными вентиляторными установками, задача управления становится на порядок сложнее. В этой ситуации кроме определения недостающего/избыточного расхода воздуха в сети необходимо определить, за счет регулирования какого именно вентилятора можно добиться требуемого расхода воздуха на регуляторах наиболее эффективным образом, а для этого, в свою очередь, необходимо знать, на каком именно регуляторе достигается максимальный недостаток/минимальный избыток воздуха.

Перед тем, как сформулировать правила выбора вентиляторной установки для регулирования на текущем шаге управления, введем некоторые определения.

Под зоной влияния конкретного вентилятора будем понимать совокупность регуляторов, на изменение расхода воздуха на которых наибольшее влияние оказывает изменение расхода воздуха на данном вентиляторе в данный момент времени.

Пусть $Z_{j}-$ зона влияния вентилятора $F_{j}$ : 


$$
Z_{j}=\left\{D_{i}\right\}_{i=1 . . N} \mid \forall j: \frac{\Delta Q_{D_{i}}}{\Delta Q_{F_{j}}} \geq \frac{\Delta Q_{D_{i}}}{\Delta Q_{F_{k}}}, k \neq j, k=1 . . K
$$

где $D_{i}-i$-ый регулятор вентиляционной сети, $\Delta Q$ - изменение расхода воздуха на вентиляторах и регуляторах.

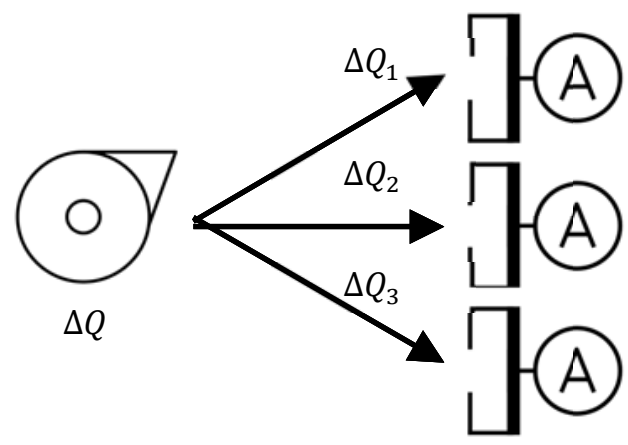

\begin{tabular}{|l|c|c|c|}
\hline & $\mathrm{D}_{\mathbf{1}}$ & $\boldsymbol{D}_{\mathbf{2}}$ & $\boldsymbol{D}_{\mathbf{3}}$ \\
\hline $\mathrm{F}_{\mathbf{1}}$ & 0,55 & 0,15 & 0,3 \\
\hline $\boldsymbol{F}_{\mathbf{2}}$ & 0,2 & $\mathbf{0 , 3}$ & $\mathbf{0 , 5}$ \\
\hline $\mathrm{F}_{3}$ & 0,7 & 0,1 & 0,2 \\
\hline
\end{tabular}

Рис. 1. Иллюстрация определения зоны влияния вентилятора на текущем шаге управления

Для определения зоны влияния необходимо после каждого изменения частоты вентилятора рассчитать, в каких пропорциях распределилось изменение расхода по всем регуляторам (строки таблицы на рис. 1), а затем для каждого регулятора определить максимальное значение доли (столбцы таблицы на рис. 1). Очевидно, что зона влияния конкретного вентилятора является плавающей, т.е. может измениться на любом шаге управления.

Под целевым регулятором будем понимать регулятор, на котором достигается максимальный недостаток (при общем недостатке) или минимальный перерасход (при общем избытке) воздуха в сети.

Пусть

$D=\left\{D_{i}\right\}$ - множество регуляторов,

$D^{*}$ - целевой регулятор,

$\Delta Q$ - общий недостаток/избыток расхода воздуха,

$\Delta Q_{i}^{*}$ - требуемый расход воздуха на регуляторе $D_{i}$,

$\Delta Q_{i}-$ фактический расход воздуха на регуляторе $D_{i}$

$$
D^{*}=D_{i} \mid \Delta Q_{i}=\left\{\begin{array}{l}
\min _{i: Q_{i} \geq Q_{i}^{*}}\left(Q_{i}-Q_{i}^{*}\right), \Delta Q \geq 0 \\
\max _{i: Q_{i}<Q_{i}^{*}}\left(Q_{i}^{*}-Q_{i}\right), \Delta Q<0
\end{array}\right.
$$

Определим следующие правила для определения степени изменения частоты вращения конкретной вентиляторной установки на текущем шаге управления.

1. В зоне влияния вентилятора хотя бы один регулятор полностью открыт.

2. Приращение частоты вращения пропорционально степени влияния на целевой регулятор.

3. При совокупном недостатке воздуха в большей степени увеличивается частота вращения вентилятора, у которого текущая частота меньше. При совокупном избытке воздухе в большей степени уменьшается частота вращения вентилятора, у которого текущая частота больше.

Первое правило является обобщением аналогичного правила для случая одной вентиляторной установки и не приводит к принципиальному изменению логики 
управления (за тем лишь исключением, что необходимо на каждом шаге пересчитывать зоны влияния вентиляторов).

Второе и третье правила в общем случае могут вступать в противоречие друг с другом, поэтому алгоритм управления должен уметь принять взвешенное решение относительно двух указанных критериев.

С этой целью в базе правил нечеткого вывода для управления вентиляторной установкой [6] при определении входной переменной отклонения скорости воздуха перейдем от нечетких множеств первого порядка к нечетким множествам второго порядка интервального типа [4].

Пусть

$\theta$ - текущая степень проветривания целевого регулятора,

$\rho$ - отношение текущей частоты к максимальной.

Тогда верхняя функция принадлежности для множеств переменной отклонения скорости воздуха будет вычисляться как

$$
\begin{gathered}
\max (\theta, \rho), \text { для термов } P, P Z \\
\max (\theta, 1-\rho), \text { для термов } N, N Z .
\end{gathered}
$$

Нижняя функция принадлежности для множеств переменной отклонения скорости воздуха будет вычисляться как

$$
\min (\theta, \rho) \text {, для термов } P, P Z
$$

$$
\min (\theta, 1-\rho) \text {, для термов } N, N Z \text {. }
$$

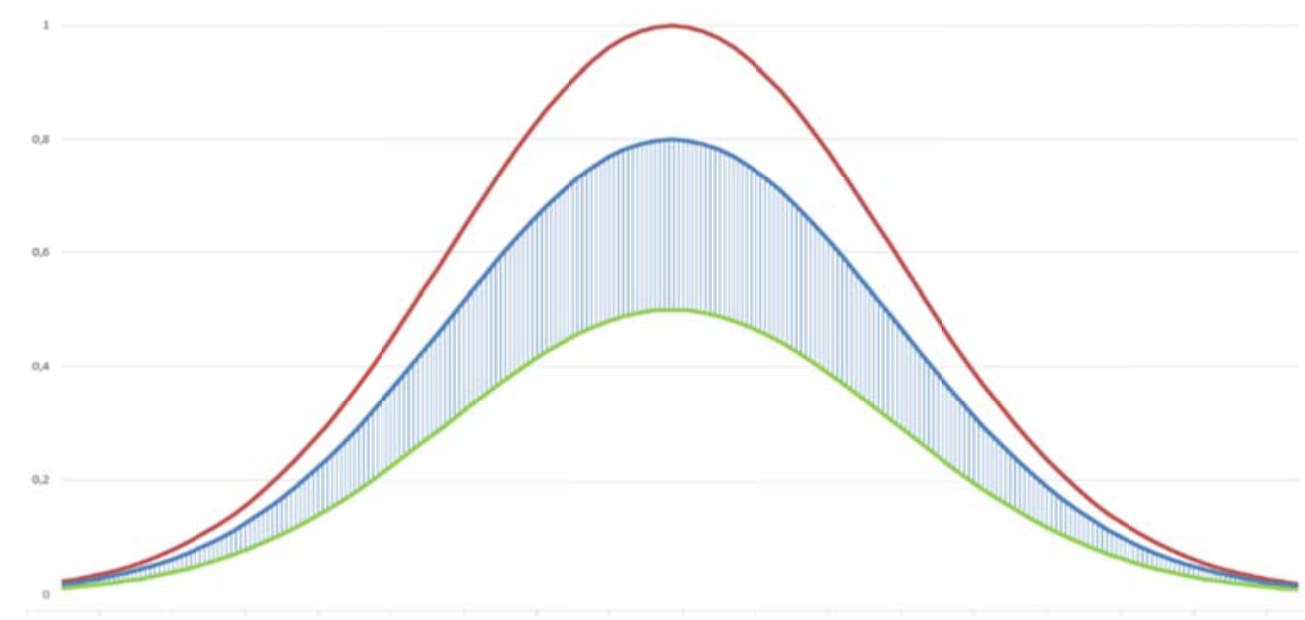

Рис. 2. Общий вид функции принадлежности для термов переменной отклонения скорости воздуха

На рис. 2 приведен общий вид получаемого следа неопределенности для множеств переменной отклонения скорости воздуха.

Термы второй входной переменной (минимальный угол открытия регулятора в зоне влияния) и выходной переменной (приращение частоты вращения) фактически остаются множествами первого порядка (у которых верхняя и нижняя функции принадлежности совпадают).

Управление регуляторами при этом остается без изменения (на основе нечеткой логики первого порядка).

Результаты моделирования (рис. 3) демонстрируют, что управление на основе множеств второго порядка дает гораздо более устойчивый результат, чем 
использование множеств первого порядка (в этом случае предполагается, что мы используем некоторый рассчитанный коэффициент для учета одновременно степени влияния и текущей частоты).

Кроме устойчивости управления, преимуществом изложенного подхода является также отсутствие необходимости в предварительных калибровочных расчетах и информации о топологии вентиляционной сети.
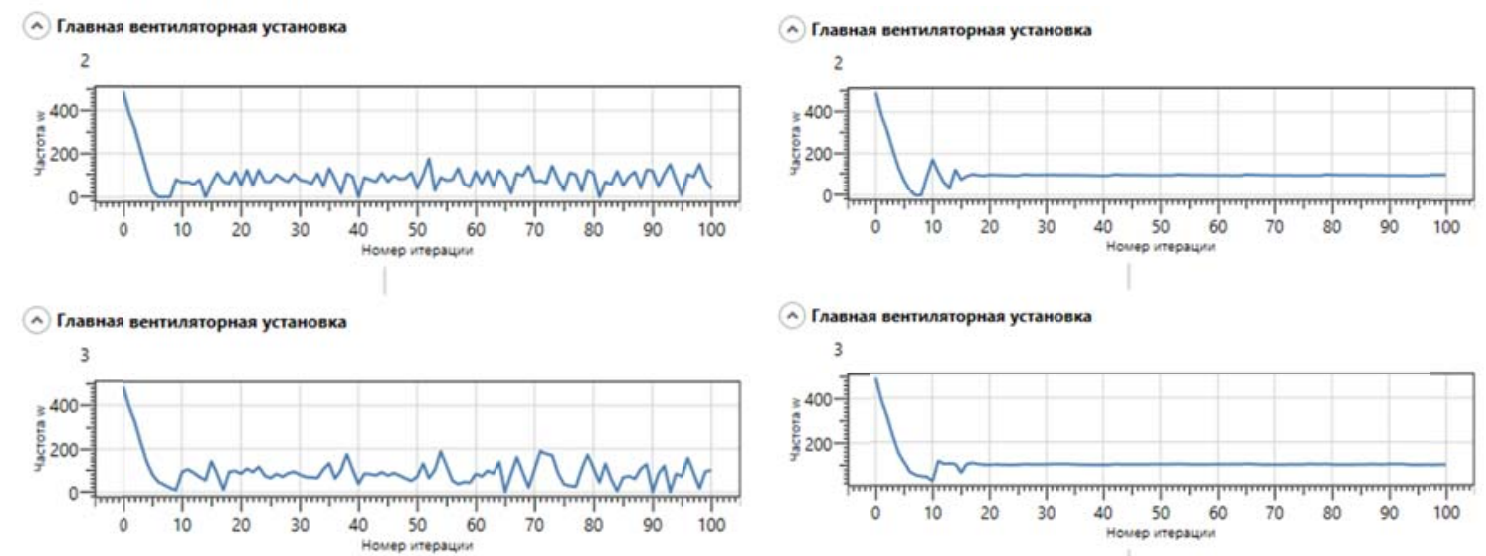

Рис. 3. Результаты моделирования работы вентиляторных установок на основе нечеткой логики первого порядка (слева) и нечеткой логики второго порядка (справа)

\section{БИБЛИОГРАФИЧЕСКИЙ СПИСОК}

1. Hardcastle S., Kocsis C., Lacroix R. Strategic mine ventilation control: a source of potential energy savings // Proceedings of Montreal Energy \& Mines, April 29-May 2. - Montreal, 2007. - P. 255-263.

2. Kashnikov A., Levin L. Applying machine learning techniques to mine ventilation control systems. // Proceedings of 2017 XX IEEE International Conference on Soft Computing and Measurements (SCM). May 24-26, 2017. - Saint Petersburg, 2017. - DOI: 10.1109/SCM.2017.7970595.

3. Levin L.Yu., Semin M. A. Conception of automated mine ventilation control system and its implementation on Belarussian potash mines // Proceedings of the $16^{\text {th }}$ North American Mine Ventilation Symposium. Colorado, 2017. - P. 17.1-17.8.

4. Mendel J.M. On the importance of interval sets in type-2 fuzzy logic systems: Proceedings Joint $9^{\text {th }}$ IFSA World Congress and $20^{\text {th }}$ NAFIPS International Conference, Canada, Jul. 25-28, 2001 . - Vancouver, 2001. P. 1647-1652. - DOI: 10.1109/NAFIPS.2001.943798.

5. Кашников А.В. Динамический расчет количества воздуха в условиях применения систем автоматического управления проветриванием рудников // Стратегия и процессы освоения георесурсов: сб. науч. тр. Вып. 14 / ГИ УрО РАН. - Пермь, 2016. - С. 259-260.

6. Кашников А.В. Применение нечеткой логики при реализации систем автоматического управления проветриванием // Стратегия и процессы освоения георесурсов: сб. науч. тр. Вып. 16 / ГИ УрО РАН. Пермь, 2018. - C. 296-299. DOI: 10.7242/gdsp.2018.16.79.

7. Круглов Ю.В., Левин Л.Ю. Основы построения оптимальных систем автоматического управления проветриванием подземных рудников // Изв. ТулГУ. Науки и Земле. - 2010. - Вып. 2. - С. 104-109.

8. Круглов Ю.В., Семин М.А. Совершенствование алгоритма оптимального управления проветриванием вентиляционных сетей сложной топологии // Вестн. ПНИПУ: Геология. Нефтегазовое и горное дело. - 2013. - № 9.- C. 106-115. 